\title{
MÉTODOS SINTÉTICOS PARA PREPARAÇÃO DE 2,2'-BIPIRIDINAS SUBSTITUÍDAS
}

Claudio Luis Donnici*, Ione Maria Ferreira de Oliveira, Eliane Sílvia Codo Temba e Maurício Costa Rogério de Castro Departamento de Química, Instituto de Ciências Exatas, Universidade Federal de Minas Gerais, Av. Antonio Carlos, 6627, 31270-901 Belo Horizonte - MG

Recebido em 13/7/01; aceito em 28/10/01

SYNTHETIC METHODS FOR PREPARATION OF SUBSTITUTED 2,2'-BIPYRIDINES. The 2,2'-bipyridine has been entitled as the most widely used ligand. Nowadays there is a large variety of known molecules comprising at least two 2,2'-bipyridine units and the number of applications in many areas such as catalysis, new materials, optoeletronics and electrochemistry have increased very much in the past decades. Nevertheless, there is no article that gives an overview of the main synthetic methods for obtaining the substituted 2,2'-bipyridines, generally non available. This article presents a synthetic discussion about the three different methods (coupling reaction, ciclo-functionalization and functionalization of the heteroaromatic rings of 2,2'-bipyridine) for preparing these heterocyclic compounds and also provides a practical and fundamental guide, for obtaining more than eighty different symmetric and unsymmetrical substituted 2,2'-bipyridines, shown in a table with the corresponding references.

Keywords: 2,2'-bipyridines; synthesis; coupling; functionalization.

\section{INTRODUÇÃO}

As bipiridinas, ou dipiridinas, são dímeros da piridina que podem ser obtidos como seis isômeros diferentes (Figura 1): 2,2'-bipiridina (1), 2,3'-bipiridina (1a), 3,3'-bipiridina (1b), 2,4'-bipiridina (1c), 4,4'bipiridina (1d) e a 3,4'-bipiridina (1e) ${ }^{1}$. Estes dímeros são sólidos cristalinos, com exceção de $\mathbf{1 b}$ que é líquido, e são obtidos, em geral, a partir da dimerização da piridina. Apenas o isômero 1a pode ser obtido de fonte natural, a fermentação de folhas de tabaco ${ }^{1 \mathrm{a}}$. As bipiridinas são, também, pouco polares e insolúveis em água e, devido aos pares de elétrons não-ligantes dos átomos de nitrogênio nos anéis heteroaromáticos, têm sido muito usadas como ligantes para formação de complexos com as mais variadas aplicações².<smiles>c1ccc(-c2ccccn2)nc1</smiles>

1<smiles>c1ccc(-c2ccncc2)nc1</smiles>

1c<smiles>c1ccc(-c2cccnc2)nc1</smiles><smiles>c1cc(-c2ccncc2)ccn1</smiles>

1d<smiles>c1cncc(-c2cccnc2)c1</smiles>

1b<smiles>c1cncc(-c2ccncc2)c1</smiles>

1e
Figura 1. Estruturas dos isômeros da bipiridina

A 2,2'-bipiridina (1), com um maior poder quelante, é certamente o mais estudado destes isômeros e um dos ligantes conhecidos mais investigados e utilizados, como se pode verificar pelas quase cinco mil citações encontradas em consulta a banco de dados disponível pela Internet ${ }^{3}$ e em extenso artigo de revisão publicado recentemente, justamente titulando a bipiridina como o ligante mais usado $^{4}$. Neste artigo, Hosseini, Katz e Kaes apresentam uma vasta revisão sobre a diversidade de moléculas com ao menos duas unida-

\footnotetext{
*e-mail: cdonnici@dedalus.lcc.ufmg.br
}

des 2,2'-bipiridínicas sintetizadas e suas correspondentes propriedades e aplicações descritas que vão desde propriedades catalíticas, eletroquímicas, luminescentes e condutoras de eletricidade, estocagem de energia solar, foto- e opto-eletrônica até estudos de interação com o DNA, reconhecimento molecular quiral e outras aplicações tecnológicas. Cabe ressaltar ainda que o interesse em 2,2'-bipiridinas e seus complexos metálicos tem aumentado muito pelo fato de que tais compostos têm apresentado, como já relatado, interação com o DNA e atividade anti-tumoral ${ }^{3}$. Apesar do citado artigo de Hosseini e col. ${ }^{4}$ reportar mais de quatrocentas estruturas bipiridínicas, complexas e até poliméricas não se tem na literatura uma visão sintética mais aplicada e objetiva sobre a obtenção de 2,2'-bipiridinas substituídas. Estes compostos, geralmente, não são comerciais e são o insumo fundamental para muitos pesquisadores nas áreas de química inorgânica e outras, além de reagente de partida para a obtenção de quaisquer outros derivados bipiridínicos.

O presente artigo mostra os principais métodos sintéticos para obtenção de 2,2'-bipiridinas substituídas, visando fornecer um guia fundamental sobre a síntese destes compostos bicíclicos heteroaromáticos nitrogenados de tão grande interesse.

\section{MÉTODOS SINTÉTICOS PARA OBTENÇÃO DE 2,2'- BIPIRIDINAS}

De fato, o grande interesse na aplicabilidade das 2,2'-bipiridinas substituídas diversas tem levado muitos pesquisadores a investigarem novas e melhores rotas sintéticas para a sua preparação. A síntese de 2,2'-bipiridinas substituídas pode ser efetuada a partir de três rotas principais descritas através das análises retrossintéticas descritas na Figura 2: i) por reações de acoplamento das piridinas correspondentes; ii) por reações de ciclização de precursores 1,5- ou a,e-dicarbonílicos apropriados; iii) por funcionalização através de reações de substituição eletrofílicas ou nucleofílicas do anel da 2,2'-bipiridina (1).

\section{Obtenção de 2,2'-bipiridinas por reações de acoplamento}

O método clássico para a obtenção de bipiridinas é através de reações de acoplamento das piridinas correspondentes. A primeira sínte- 


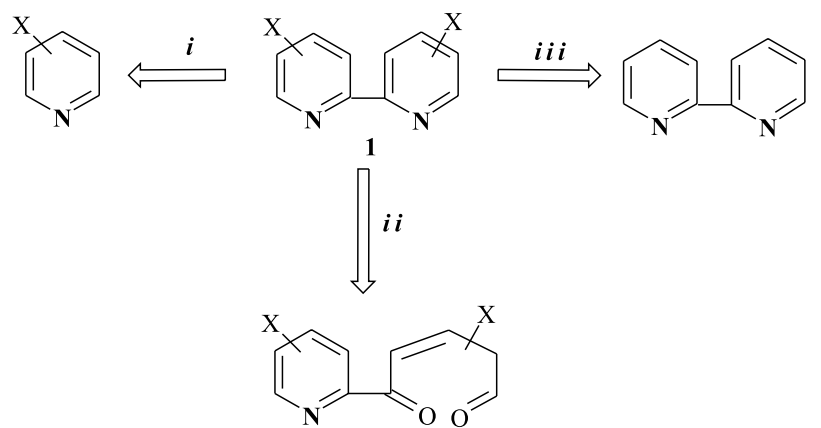

Figura 2. Análises retrossintéticas para obtenção de 2,2'-bipiridinas substituídas

se da 2,2'- bipiridina (1) foi descrita por F. Blau ${ }^{5}$ a partir da decomposição por aquecimento do sal de cobre do ácido nicotínico. Contudo, os métodos gerais descritos na literatura para a obtenção da 2,2'bipiridina (1), usam a reação de Chichibabin ${ }^{6}$ modificada: a preparação da bipiridina a partir da reação da piridina com sódio metálico, ou amideto de sódio em amônia líquida. Contudo, estes métodos não são seletivos e mesmo as outras bipiridinas isoméricas 1a, 1b e 1d (Figura 1) podem ser obtidas, além da própria 2-amino-piridina, produto usual esperado neste tipo de reação ${ }^{1}$. O melhor rendimento (43\%) para a obtenção de 1, só é alcançado partindo-se da reação de acoplamento de piridina-N-óxido com cloreto de amônio e sódio em amônia ${ }^{7}$.

A metodologia de acoplamento de piridinas para a obtenção de 2,2'-bipiridinas simétricas foi melhorada pela utilização da reação de Ullmann ${ }^{8}$, o acoplamento de derivados halogenados aromáticos por tratamento com cobre à alta temperatura. Assim, 1 pode ser obtida, em $60 \%$ de rendimento, a partir da 2-bromo-piridina ${ }^{9}$, ou em rendimentos ainda melhores através de uma mistura de cloreto de sódio e cobre metálico, na proporção de 1,5:1.

O método de Ullmann possibilitou também a preparação de diversas 2,2'-bipiridinas substituídas $\mathbf{2}$, em bom rendimento, a partir da reação de bromo-piridinas correspondentes ${ }^{10-12} \mathbf{3}$ (Esquema 1). As outras halo-piridinas, bem como a substituição de cobre por sódio mostraramse menos eficientes. Entretanto, vale ressaltar a obtenção da 2,2'-bipiridina (1) a partir da reação de piridina com cloreto férrico ${ }^{13}$ e a utilização de brometo férrico e bromo com luz para dimerização de piridina substituida ${ }^{13}$. O uso de cloreto férrico e de níquel de Raney ${ }^{14}$, bem como de catalisador de níquel e alumínio ${ }^{14}$ e iodo ${ }^{11,16}$ são métodos que usam de desidrogenação catalítica. Também foram relatadas, mais recentemente, modificações na desidrogenação catalítica com o uso de paládio ${ }^{17}$; contudo, no geral, as variações deste método precisam de longos tempos de reação e levam a baixos rendimentos. Os estudos de Sasse e colaboradores ${ }^{14}$ merecem destaque pois, prepararam vários derivados bipiridínicos simétricos $\mathbf{3}$ por dimerização das picolinas, colidinas, fenil-piridinas e outras piridinas substituídas correspondentes.

$$
\begin{aligned}
& \text { reação de } \\
& \mathrm{Y}=\text { a) } 5-\mathrm{Br}, \mathrm{b}) 5-\mathrm{Cl}, \mathrm{c}) 5-\mathrm{NO}_{2}{ }^{10} \\
& \mathrm{Y}=\mathrm{d}) 3-\mathrm{Me}, \mathrm{e}) 4-\mathrm{Me}, \mathrm{f}) 5-\mathrm{Me}^{10,14} \\
& \left.\mathrm{Y}=\mathrm{g}) 6-\mathrm{Me}^{11,14} ; \mathrm{h}\right) 4-\mathrm{Et}^{12,14} ; \text { i) } 4-\mathrm{C}_{6} \mathrm{H}_{5}{ }^{12} \\
& \mathrm{Y}=\text { j) } 3,5-(\mathrm{OEt})_{2}-6-\mathrm{Br}^{13} \\
& \mathrm{Y}=\text { l) } 5-\mathrm{Et}, \mathrm{m}) 5-\mathrm{CO}_{2} \mathrm{H}, \text { o) } 5-\mathrm{COC}_{6} \mathrm{H}_{5}{ }^{14}
\end{aligned}
$$

Newkome e Hager ${ }^{18}$ haviam relatado em 1978 que derivados fosfinóxidos do tipo $\mathbf{4}$ formados a partir da litiação de halo-piridinas seguida de fosforilação e oxidação levam, por decomposição térmica, aos derivados bipiridínicos correspondentes (Esquema 2). De fato, Oae, Uchida e Kozawa ${ }^{19}$ reportaram posteriormente, em 1989, um acoplamento análogo com sal de fosfônio 5 como agente de acoplamento (Esquema 3). Em 1995, Oae e colaboradores ${ }^{20}$ desenvolveram uma metodologia mais satisfatória para a formação de 2,2'bipiridina (1) através de acoplamento de piridina. Utilizando cloreto de tionila como agente de acoplamento, os autores observaram que os sais de lítio formados pela litiação de a-bromopiridina (2) e a reação com cloreto de tionila conduzem à formação de 1, em $55 \%$ de rendimento (Esquema 4). O acoplamento também ocorre com tricloreto de fósforo $(50 \%)$ e oxicloreto de fósforo $(51 \%)$.

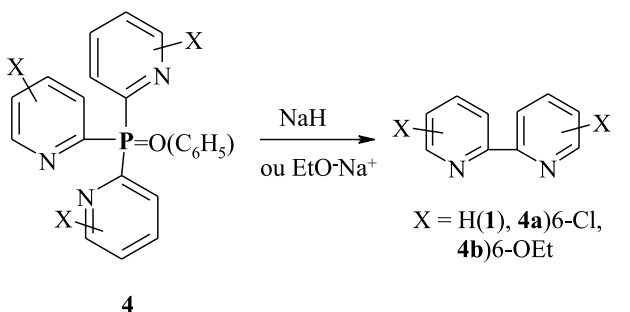

Esquema 2

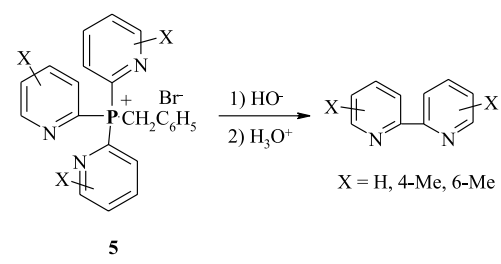

Esquema 3

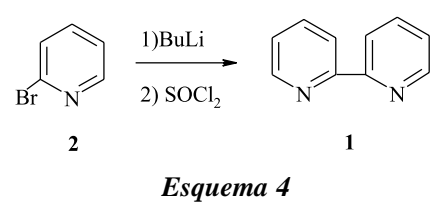

Apesar do uso moderno e amplo de compostos organometálicos em síntese orgânica, há poucos relatos específicos para a síntese de 2,2'-bipiridinas com o uso destes. Holm, Wagner e Parks ${ }^{21}$ e Garber e Rillema ${ }^{22}$ descreveram a obtenção de 6-bromo-2,2'-bipiridina (6a) e de 6,6'-dibromo-2,2'-bipiridina (6b) a partir da litiação de 2,6dibromo-piridina (7) na presença de sais de cobre (II) e (I), respectivamente, (Esquema 5) o que sugere a formação de um intermediário tipo organocuprato.

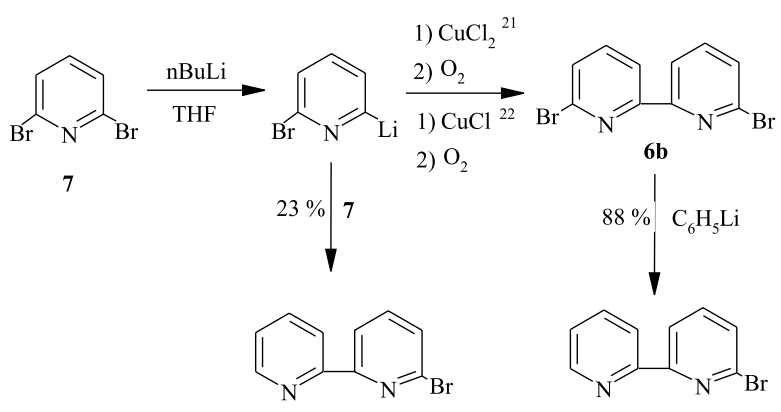

$6 a$

6a 
Em 1999, Leadbeater e Resouly ${ }^{23}$ relataram o acoplamento de halo-piridinas substituídas às correspondentes bipiridinas $\mathbf{8}$, em rendimentos de cerca de $70 \%$, pelo uso catalítico de biscarbonilbistetrafenilfosfina de níquel zero em dimetilformamida a $70{ }^{\circ} \mathrm{C}$ (Esquema 6).

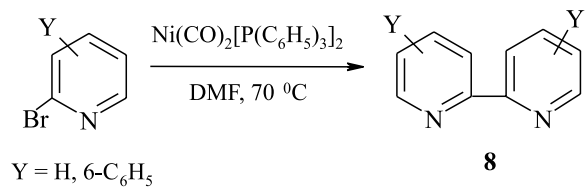

\section{Esquema 6}

Schubert e colaboradores ${ }^{24 a}$ descreveram, recentemente, a obtenção de 2,2'-bipiridinas em bons rendimentos, utilizando compostos organometálicos de estanho 9a, formados a partir da transmetalação (THF, a $-78^{\circ} \mathrm{C}$ ) da lítio-piridina correspondente com cloreto de tributil-estanho e a utilização de catalisadores de paládio (Esquema 7a). A obtenção da terpiridina funcionalizada 10, em excelente rendimento, foi relatada pela reação do composto organoesta nho derivado formado com 2,6-dibromo-piridina 7. Panetta e col. ${ }^{24 b}$ descreveram a formação de derivados organometálicos piridínicos de estanho 9b para a obtenção de derivados 2,2'-bipiridínicos carbometóxi substituídos diversos 11a-d e outros derivados (Esquema $7 b)$.

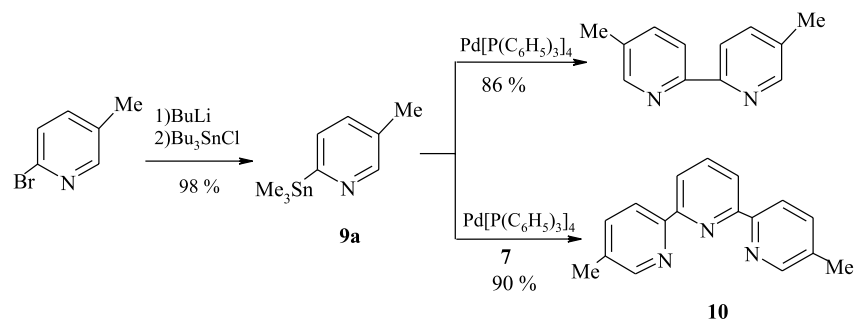

Esquema $7 a$

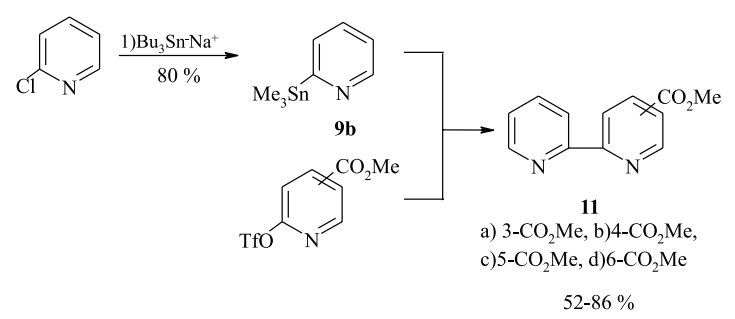

Esquema $7 b$

Mesmo com este exemplo, observa-se que a metodologia moderna de acoplamento através de compostos organometálicos para síntese de 2,2'-bipiridinas simétricas ainda é pouco utilizada.

Fraser e colaboradores ${ }^{25}$, em 1998, elaboraram uma eficiente estratégia para síntese de 2,2'-bipiridinas não-simétricas metílicas por acoplamento cruzado. Nesta nova metodologia primeiramente transforma-se a $\alpha$-hidróxi-piridina substituída 12a no triflato correspondente 12, o qual é ,então, submetido à reação com o organozinco formado de 2-bromo-piridina (12b), na presença catalítica de bis-dibenzilidenoacetona 13 e trifenilfosfina (TFF), obtendo-se a metil-2,2'-bipiridina não-simétrica correspondente 14, em bons rendimentos (Esquema 8).

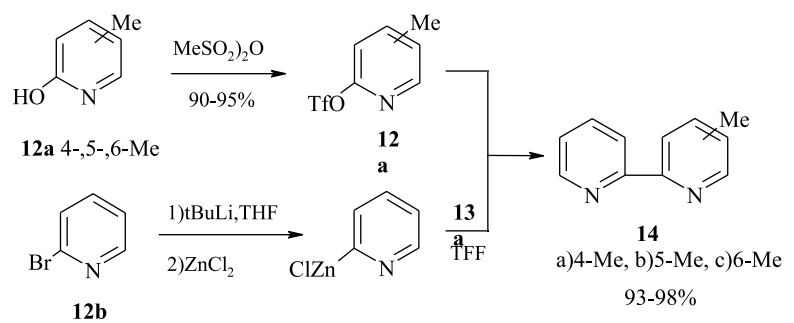

Esquema 8

Navarro e col. ${ }^{26}$ também relataram, em 2000, a preparação de 6,6-dimetil-2,2-bipiridina (3g) pelo acoplamento de 2-bromo-picolina (15) através de método eletrossintético catalisado por sais de níquel e pela formação intermediária de complexo de bisbrometo-bipiridina de níquel (II)(rendimento de $76 \%$ ); ou por uso de acetato de paládio (74 \%), em uma reação de Ullmann modificada. Neste ano, os mesmos autores estenderam a metodologia para a obtenção de 3,3'- (3d), 4,4'-(3e) e 5,5'-dimetil-2,2'-bipiridinas (3f $)^{27}$.

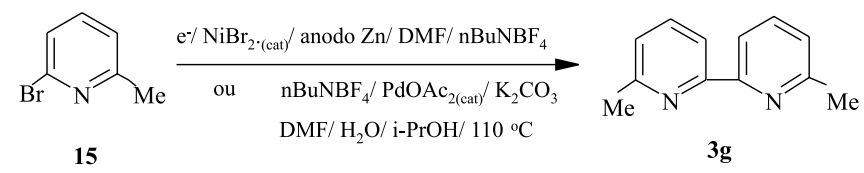

Esquema 9

Os exemplos acima demonstram que o método de acoplamento é satisfatório quando há disponibilidade da piridina substituída de partida, apesar de que geralmente não é um método muito prático, pelo baixo rendimento obtido, exceto com o uso dos métodos modernos de acoplamento com organometálicos, onde mesmo bipiridinas não-simétricas podem ser sintetizadas com bons rendimentos.

\section{Obtenção de 2,2'-bipiridinas por reações de ciclo- funcionalização}

Os métodos clássicos para a obtenção de anéis piridínicos geralmente envolvem a ciclização de compostos 1,5-dicarbonílicos, ou derivados, com amônia; métodos de cicloadição e outros ${ }^{28}$. Contudo, para a síntese de bipiridinas, estas metodologias específicas foram empregadas a partir de métodos adaptados; somente o estudo de Potts e Winslow ${ }^{29 a}$, de 1985, é destacável. Neste trabalho sintetizouse a 2,2'-bipiridina não-simétrica 4,6-dissubstituída 15 - $(\mathrm{R}=\mathrm{Me}$, $\left.\mathrm{R}^{1}=\mathrm{SMe}, \mathrm{SEt}\right)$ e outros derivados por reações de oxidação e dessulfurização partindo-se do precursor 3,3-bis-(metil- ou etilsulfanil)-1-(2-piridinil)-2-propen-1-ona (16) através da ciclização do intermediário 1,5- (ou $\left.\alpha, \varepsilon^{-}\right)$dicarbonílico $\mathbf{1 7}$, formado pela condensação com o enolato potássico da acetona 18. A condensação do precursor 17 com o enolato do acetaldeído 19 gera bipiridinas não-simétricas 4,5-disubstituídas 20 e outros derivados por dessulfurização. Outros derivados heteroaromáticos do tipo 21, também foram obtidos a partir da ciclo-funcionalização do precursor furânico 22, com o enolato potássico da 2-acetil-piridina 23. O derivado terc-butil-bipiridinil-substituído $\mathbf{2 4}$ também foi obtido a partir da reação do ceteno-tiocetal 25 com o enolato 23 (Esquema 10). Apesar da complexidade dos precursores usados, este método sintético apresenta bons rendimentos globais, cerca de 30 até $90 \%$, e permite a preparação de bipiridinas de padrão de substituição diferentes. Posteriormente, Potts e colaboradores ${ }^{29 b}$ usaram esta metodologia para o desenho racional de 2,6-oligopiridinas. 


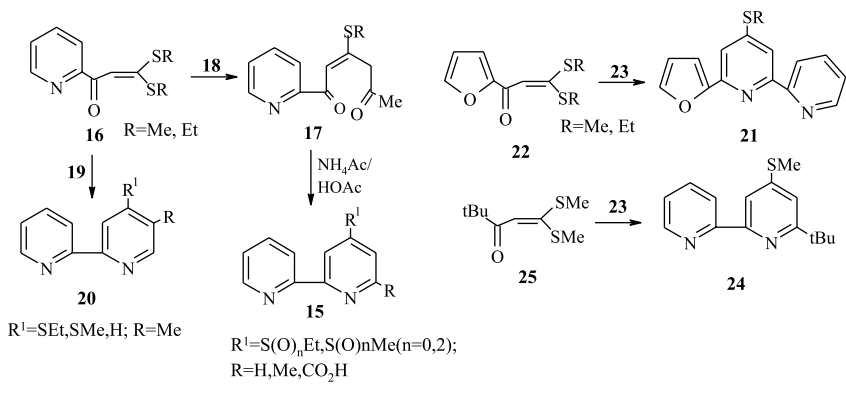

Esquema 10

\section{Obtenção de 2,2'-bipiridinas substituídas por funcionalização da 2,2'- bipiridina}

A reatividade e regiosseletividade da 2,2'-bipiridina (1), apesar de não muito estudadas, são similares às da piridina ${ }^{28}$, o que possibilita a obtenção análoga de derivados substituídos por reações de substituição eletrofílica ou nucleofílica aromáticas. A reação de substituição eletrofílica direta da bipiridina é tão difícil quanto a da piridina, devido à baixa densidade eletrônica do anel heteroaromático, com o efeito dirigente levando à obtenção de derivados 3- (ou $\beta$-) ou 3,3'substituídos $^{28}$. Porém, a bromação da 2,2'-bipiridina (1) (à uma temperatura elevada, de $500{ }^{\circ} \mathrm{C}$ ) como já observado nos trabalhos pioneiros de Burstall ${ }^{16,30}$ de 1934 a 1938, conduzia segundo os autores da época, à 6,6'-dibromo-2,2'-bipiridina e também à 6-bromo-2,2'bipiridina (Esquema 11). Romero e Ziessel ${ }^{31}$, em 1995, refizeram estes estudos por dois métodos, um análogo ao de Burstall e um outro descarboxilativo, e comprovaram que a bromação de $\mathbf{1}$ leva na verdade aos derivados esperados 5-bromo-(ou $\beta$-) 3a e 5,5'-dibromosubstituídos 26. Burstall também descreveu, no mesmo trabalho citado $^{30}$, a obtenção dos correspondentes 5-amino- e 5,5' -diaminoderivados 27 , por reação com amideto de sódio. A partir destes derivados vários outros sistemas bipiridínicos podem ser obtidos ${ }^{4}$. Por exemplo, as nitrilas $\mathbf{2 8}$, por reação com cianeto de cobre e os ácidos carboxílicos análogos $\mathbf{3 m}$, por hidrólise ácida destes (Esquema 11).
Vários pesquisadores estudaram a síntese de outros derivados 2,2'-bipiridínicos, ressaltando-se o trabalho de Case $^{9}$ que descreveu a obtenção de derivados carboxílicos bipiridínicos 3m e 29 a partir da oxidação das dimetil-bipiridinas 3e e $3 \mathbf{f}$ correspondentes com permanganato de potássio, que é o reagente usual para este tipo de oxidação (Esquema 12). Donnici e col..$^{32}$, em 1998, relataram o uso de dicromato de potássio em meio ácido para a obtenção de 29, em melhores rendimentos (85\%). Cabe lembrar que com a obtenção dos derivados bipiridínicos carboxílicos podem-se obter posteriormente diversos derivados carboxílicos $\mathbf{3 0}^{32-34}$. No artigo de Hosseini, Katz e Kaes ${ }^{4}$ pode-se verificar diversas outras derivatizações de bipiridinas carboxílicas. Outras reações de $\alpha$-funcionalização de grupos alquílicos em bipiridinas serão discutidas posteriormente.

Em 1955, quando Haginiwa ${ }^{35}$ preparou a N,N'-dióxido-2,2'bipiridina (31), pela primeira vez, a partir da oxidação da 2,2'bipiridina (1) com ácido peracético gerado "in situ” (Esquema 13) foi possível a preparação mais eficiente de derivados bipiridínicos 4,4'-e 2,2'-dissubstituídos. O derivado N,N'-dióxido 31 é bem mais reativo que a bipiridina apresentando efeito 4- (ou $\gamma$-) dirigente em reações eletrofílicas ${ }^{28}$, e 2- (ou $\alpha$-) dirigente em substituições nucleofílicas (Esquema 13). Por exemplo, a partir do N,N'-dióxido 31, o derivado 6,6'-diciano-2,2'-bipiridina $(32)^{36}$ pode ser obtido facilmente, por reação de substituição nucleofílica específica, com cianeto de potássio e cloreto de benzoíla ou por reação com cianofosfonato de etila ${ }^{37}$. A 4,4'-dinitro-2,2'-bipiridina (33) pode ser obtida pela redução, com tricloreto de fósforo em clorofórmio anidro, do derivado correspondente N,N'-dióxido, que pode ser obtido, em $49 \%$ de rendimento, a partir de reação de substituição eletrofílica (nitração clássica da 2,2'-bipiridina (1)) conforme descrito pelo próprio Haginiwa ${ }^{38}$; mais recentemente esta reação foi otimizada por Donnici e col. ${ }^{32}$ (75\%), pelo uso de excesso de agente nitrante. A redução da correspondente N,N'-dióxido-bipiridina para a obtenção de 33, primeiramente descrita por $\mathrm{Case}^{38}$, com baixo rendimento (10\%) em 1958, foi depois otimizada (rendimento de 93\%) por Wenkert e Woodward ${ }^{39}$, em 1983, pelo uso de excesso de tricloreto de fósforo e maior tempo reacional (Esquema 13); estes resultados foram confirmados recentemente por nosso grupo. A partir do deri-

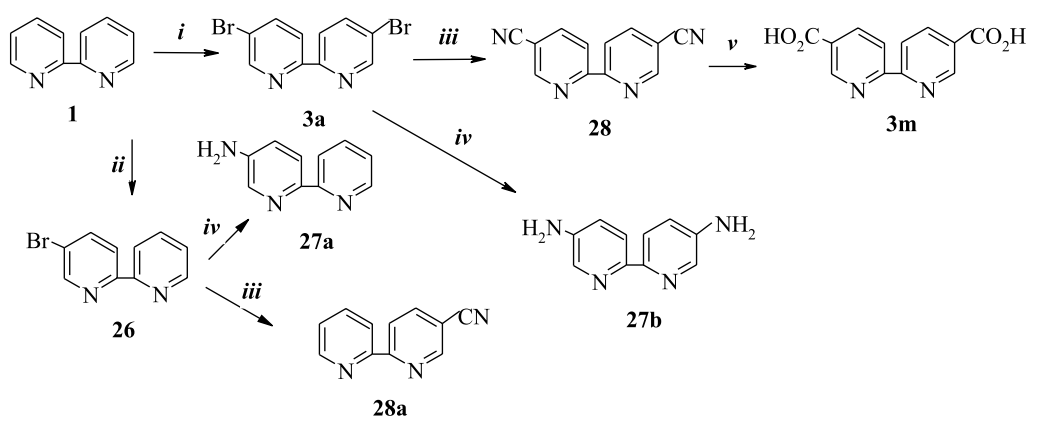

i) $\mathrm{Br}_{2} / 180{ }^{\circ} \mathrm{C}, 72 \mathrm{~h}(51 \%) ;$ ii) $\mathrm{Br}_{2} / 180{ }^{\circ} \mathrm{C}, 24 \mathrm{~h}(46 \%) ;$ iii) $\mathrm{CuCN} / 90{ }^{\circ} \mathrm{C}(40 \%) ;$ iv $\left.) \mathrm{NH}_{4} \mathrm{OH} / 220^{\circ} \mathrm{C}, 14 \mathrm{~h} ; \boldsymbol{v}\right) \mathrm{H}_{3} \mathrm{O}^{+}$

Esquema 11

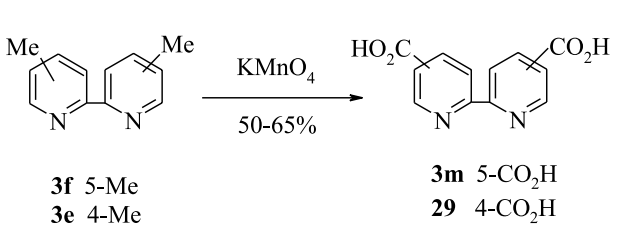

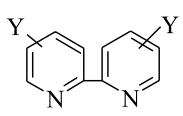

30a) $\mathrm{Y}=4-\mathrm{COCl}^{33}$

30b) $\mathrm{Y}=4-\mathrm{CO}_{2} \mathrm{R}^{33}$

30c) $\mathrm{Y}=4-\mathrm{CONHOH}^{32}$

30d) $\mathrm{Y}=4-\mathrm{COSEt}^{34}$ 
vado 4,4'-dinitrado 33, pode-se preparar por redução a respectiva 4,4'-diamina 34 e obter-se também a 4,4'-dicloro-2,2'-bipiridina (35), por reação específica com cloreto de acetila ${ }^{40} \mathrm{e}$ tricloreto de fósfo$\mathrm{ro}^{41}$, respectivamente (Esquema 13).

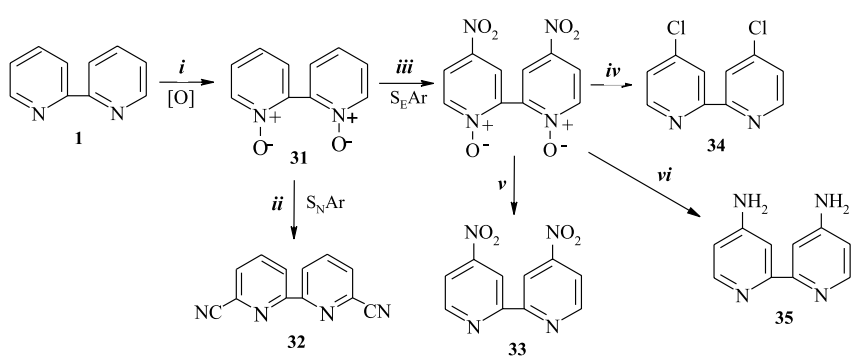

i) $\mathrm{MMPP}(75 \%)$, $\mathrm{MCPBA}(80 \%), \mathrm{MeCO}_{3} \mathrm{H}(95 \%)$;ii) $\left.\mathrm{KCN} / \mathrm{C}_{6} \mathrm{H}_{5} \mathrm{COCl}(60 \%) / \mathrm{EtO}\right)_{2} \mathrm{POCN}(85 \%)$; iii) $\mathrm{HNO}_{3} / \mathrm{H}_{2} \mathrm{SO}_{4}\left(49-75 \%\right.$; iv) $\mathrm{PCl}_{3} / \mathrm{MeCOCl}\left(68-96 \%\right.$ ); v) $\mathrm{PCl}_{3}$ ( $93 \%$ ); vi) $\mathrm{NaBH}_{4} / \mathrm{Pd}-\mathrm{C}(74 \%$ )

\section{Esquema 13}

A partir dos derivados bipiridínicos 6-monobromado 6a e 6,6'dibromado $\mathbf{6 b}$ pode-se efetuar a litiação, com butil-lítio ${ }^{21}$, seguida de reação com derivados eletrofílicos diversos e preparar outros derivados como a 6,6'-dimetil-bipiridina $\mathbf{3 g}$ e os compostos $\mathbf{3 5}$ a-c (Esquema 14).

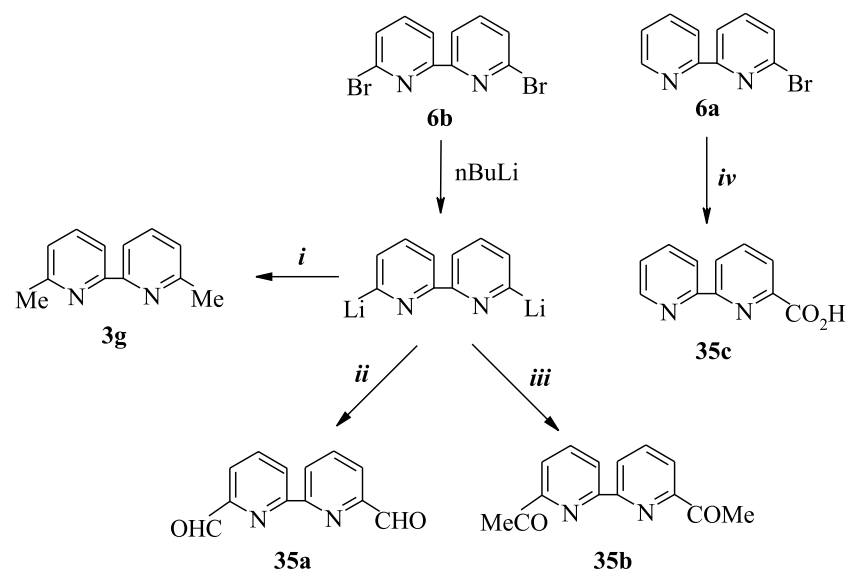

i) $\mathrm{Me}_{2} \mathrm{SO}_{4}(50 \%)$;ii) $\mathrm{HCONMe}_{2}(48 \%)$; iii) $\mathrm{MeCONMe} 2(59 \%)$; iv) $\mathrm{nBuLi} / \mathrm{CO}_{2}(50 \%)$

\section{Esquema 14}

Pode-se ainda reagir os derivados bipiridínicos halogenados com nucleófilos, como investigado para a bipiridina diclorada 34, para se conseguir a formação de diversos outros derivados como os compostos 36 a $\mathbf{a}, \mathbf{b}^{40}$ e 37 a, $\mathbf{b}^{39,42}$ (Esquema 15). Em muitos casos, devido à baixa reatividade de anéis piridínicos frente à substituição nucleofílica, faz-se a reação com o derivado N,N'-dióxidohalossubstituído e só então obtém-se a bipiridina substituída por reação de redução.

Os métodos descritos nos Esquemas 14 e 15 poderiam ser aplicados com as bipiridinas halogenadas $\mathbf{3 a - b}, \mathbf{4 a}$ e $\mathbf{6 a}$, através dos quais obter-se-iam outros derivados bipiridínicos de interesse. Existem muitos exemplos da aplicação desta metodologia sintética para a obtenção de derivados bipiridínicos diversos em artigo já mencionado ${ }^{4}$.

A síntese de derivados 2,2'-bipiridínicos não-simétricos é também de interesse mas, mesmo a obtenção de derivados 3monosubstituídos por reações de funcionalização eletrofílica da 2,2'bipiridina não é muito comum, pois não se obtém seletividade com

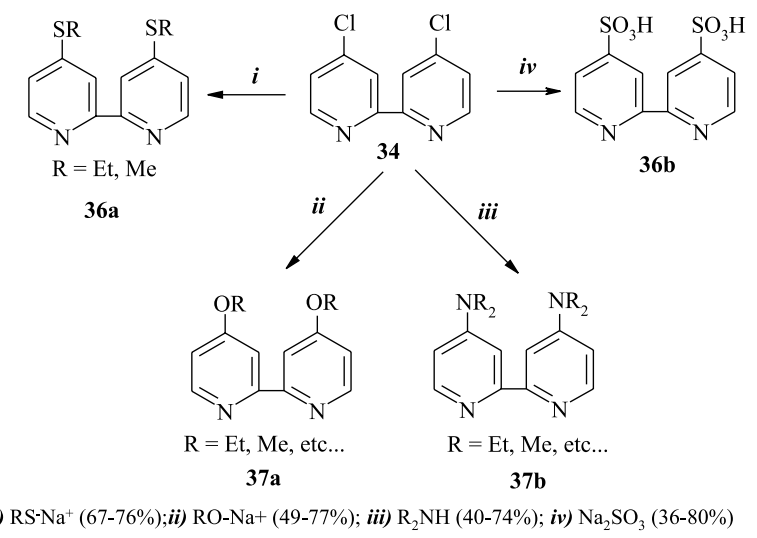

Esquema 15

exceção do caso da 5-bromo-2,2'-bipiridina (26) já relatada. Todavia, a preparação de 2,2'-bipiridinas-4-monosubstituídas pode ser conseguida satisfatoriamente a partir de reações de funcionalização, como as já descritas, com a N-óxido-2,2'-bipiridina (38) que pode ser preparada fácil e eficientemente pela oxidação seletiva de $\mathbf{1}$ ao mono-N-óxido correspondente, por ácido meta-cloro-perbenzóico $(\mathrm{MCPBA})^{39}$, ou monoperftalato de magnésio (MMPP) ${ }^{32}$ ou ácido perfluoracético gerado "in situ" ${ }^{43}$. De fato, cabe destacar as primeiras preparações de derivados bipiridínicos não-simétricos ${ }^{44} 38$ por Jones e col., através da obtenção do derivado mono-N-óxido-2,2'-bipiridina correspondente, o que induz à maior reatividade $\mathrm{e}$ às posteriores mono-funcionalizações somente no anel heteroaromático oxidado. Os autores também determinaram espectrometricamente os valores de pKa das bipiridinas estudadas. Posteriormente, Wenkert e Woodward ${ }^{42}$ prepararam outros derivados bipiridínicos não-simétricos 39. Antonini e col. ${ }^{45,46}$ também prepararam bipiridinas não-simétricas 6-ciano-substituídas $\mathbf{4 0}$ e os correspondentes derivados carbotioamídicos 41 a-g que são destacáveis por terem chamado muito a atenção pela atividade antitumoral através da inibição da síntese de DNA. A transformação de nitrila em carbotioamida pode ser efetuada em condições menos complexas e em melhor rendimento, usando-se tioacetamida em meio ácido, como descrito por Donnici e col. ${ }^{32}$.

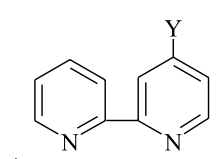

38

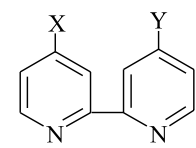

39

a) $\mathrm{X}=\mathrm{Cl}, \mathrm{Y}=\mathrm{NO}_{2}$; b) $\mathrm{X}=\mathrm{OMe}, \mathrm{Y}=\mathrm{NO}_{2}$<smiles>[X]c1ccnc(-c2cc([X])cc([Z])n2)c1</smiles>

41
$\mathrm{Y}=$ a) $\mathrm{NMe}_{2}$, b) $\left.\mathrm{NH}_{2}, \mathrm{c}\right) \mathrm{OEt}$ d)OMe, e)OBz, f)Cl, g)Br, h) $\mathrm{CONH}_{2}$, i) $\left.\mathrm{NO}_{2}, \mathrm{j}\right) \mathrm{SBz}$

$$
\begin{aligned}
& \text { a) } X=\mathrm{Y}=\mathrm{H}, \mathrm{Z}=\mathrm{CSNH}_{2} \\
& \text { b) } \mathrm{X}=\mathrm{H}, \mathrm{Y}=\mathrm{Cl}, \mathrm{Z}=\mathrm{CSNH}_{2} \\
& \text { c) } \mathrm{X}=\mathrm{NO}_{2}, \mathrm{Y}=\mathrm{H}, \mathrm{Z}=\mathrm{CSNH}_{2} \\
& \text { d) } \mathrm{X}=\mathrm{H}, \mathrm{Y}=\mathrm{OMe}, \mathrm{Z}=\mathrm{CSNH}_{2} \\
& \text { e) } \mathrm{X}=\mathrm{Y}=\mathrm{Me}, \mathrm{Z}=\mathrm{CSNH}_{2} \\
& \text { f) } \mathrm{X}=\mathrm{Y}=\mathrm{OMe}, \mathrm{Z}=\mathrm{CSNH}_{2} \\
& \text { g) } \mathrm{X}=\mathrm{Y}=\mathrm{Cl}, \mathrm{Z}=\mathrm{CSNH}_{2}
\end{aligned}
$$

É importante citar a síntese dos novos sistemas quelantes derivados de bipiridina do tipo 6,6'-bis-tiazolil-substituída 42 e o poliheteroaromático 43 a partir da bis-carbotioamida 44 descrita por Connor, Baxter e col. ${ }^{47}$ é digna de nota (Esquema 16). Diversos outros sistemas poli- cíclicos e poli-heteroaromáticos já foram sintetizados e podem ser visualizados conjuntamente no já citado artigo de Hosseini e col. ${ }^{4}$. 

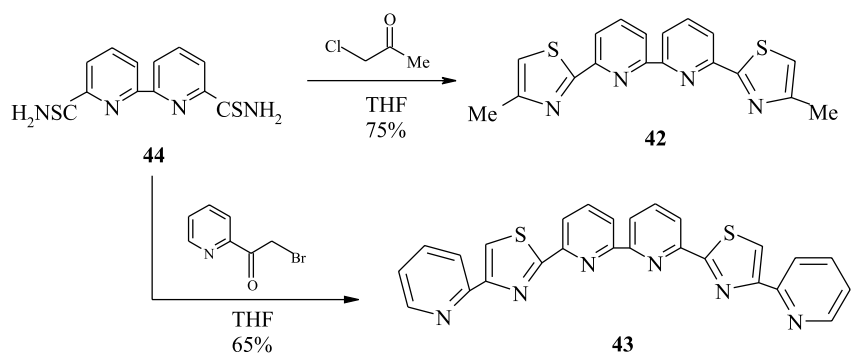

43

Esquema 16

Derivados bipiridínicos $\alpha$-metil-substituídos são também muito importantes e podem ser obtidos por diferentes métodos. A obtenção de derivados bis- $\alpha, \alpha^{\prime}$-halometil ou mono- $\alpha$-halo-2,2'bipiridínicos por halogenação radicalar com N-halo-succinimida é descrita por vários autores ${ }^{24 a, 48-50}$ porém, os rendimentos são baixos (24-60\%), a separação cromatográfị ca é díficil e há relatos sobre a irreprodutibilidade e a ineficiência deste método ${ }^{51}$. Porém, estes derivados $\alpha$-halogenados bipiridínicos são de grande importância para funcionalização lateral e construção de muitos outros derivados de interesse ${ }^{4}$. Em 1997, Fraser e col..$^{52}$ descreveram um novo método para obtenção de derivados $\alpha$-halogenados bipiridínicos 45 e $\mathbf{4 6}$, através da formação intermediária de derivados $\alpha$-sililados $\mathbf{4 7}$ seguido da reação com espécies halogenadas eletrofílicas formadas na presença de fontes de fluoreto (fluoreto de césio em DMF ou fluoreto de tetrabutil amônio em THF) (Esquema 17). A adição eletrofílica também pode ser efetuada com outros reagentes, tais como benzaldeído e haletos de alquila levando, respectivamente, a outros derivados bipiridínicos 48 e $\mathbf{4 9}^{52}$ (Esquema 17). Em extensão do método ${ }^{53}$ pode-se obter outros derivados a-substituídos 50 e 51, novos "synthons" de grande aplicabilidade.

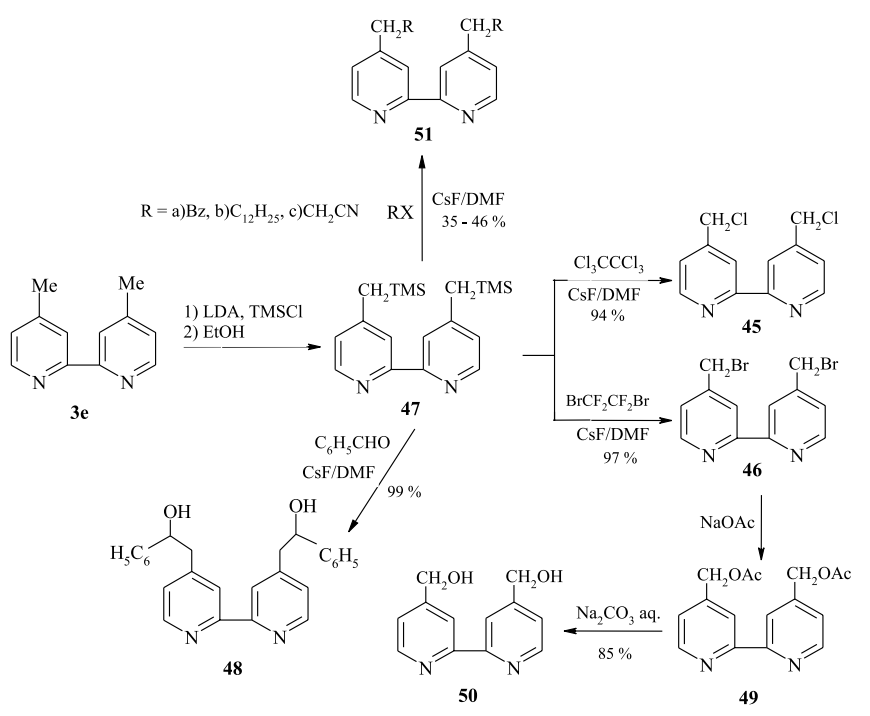

Esquema 17

É importante citar que o derivado 6,6'-bis-acetato análogo 50a pode ser obtido pela reação de N,N'-dióxido-2,2'-bipiridina (31) com anidrido acético, sob refluxo e hidrólise ${ }^{33}$. $\mathrm{O}$ derivado 6,6'-diidróxibipiridínico 50 correspondente pode ser conseguido por dois métodos: oxidação seletiva de 6,6-dimetil-bipiridina (3g) com dióxido de selênio seguida de redução, ou por hidrólise de 49 . O método de oxi- dação parcial de alquil-aromáticos com dióxido de selênio é uma alternativa interessante para $\alpha$-funcionalização de alquil-bipiridinas ${ }^{54,55}$. Por exemplo, pode-se obter seletivamente a 4-formil-2,2'-bipiridina (52) pela oxidação da 4-metil-2,2'-bipiridina (14a) ${ }^{54}$ e a 4,4'-diformil2,2'-bipiridina (53) pela oxidação da 4,4'-dimetil-2,2' -bipiridina (3e $)^{55}$ (Esquema 18). O uso sintético de 53 é evidenciado pela preparação da 4,4'-divinil-2,2'-bipiridina (54), por Ciana e col. ${ }^{55}$, a partir de reação de Wittig com 53, bem como a preparação do derivado não-simétrico 4-metil-4'-vinil-2,2'-bipiridina (55) que já tinha sido reportado anteriormente ${ }^{56}$. Estes derivados carbonílicos e insaturados são de grande potencialidade sintética pois, a presença da carbonila ou da $\alpha$ insaturação permite ampliar não só os tipos possíveis de funcionalizações como também o escopo das aplicações ${ }^{4}$.<smiles>Cc1ccnc(-c2ccccn2)c1</smiles><smiles>O=Cc1ccnc(-c2ccccn2)c1</smiles>

52<smiles>Cc1ccnc(-c2cc(C)ccn2)c1</smiles>
3 e<smiles>CS(=O)(=O)CCS(=O)(=O)O</smiles><smiles>O=Cc1ccnc(-c2cc(C=O)ccn2)c1</smiles>

53<smiles>Pc1ccccc1</smiles><smiles>C=Cc1ccnc(-c2cc(C=C)ccn2)c1</smiles>

54

Esquema 18

\section{CONCLUSÕES GERAIS SOBRE SÍNTESE DE 2,2'-BIPIRIDINAS SUBSTITUÍDAS}

\section{Métodos através de acoplamento de piridinas}

a) 2,2'-bipiridinas simétricas dissubstituídas nas posições 3,3'-, 4,4'-, 5,5'- e 6,6'- podem ser preparadas por métodos de acoplamento usuais desde que se disponha das piridinas funcionalizadas de partida. Apesar do baixo rendimento dos métodos clássicos de acoplamento, a utilização de compostos organometálicos viabiliza esta metodologia. Este campo de pesquisa é promissor para a obtenção de 2,2'-bipiridinas diversas.

b) 2,2'-bipiridinas não-simétricas podem ser obtidas por acoplamento usando-se organometálicos.

c) O método de acoplamento pode ser também particularmente usado, como única possibilidade de execução viável, para a formação de 2,2-bipiridinas-3,3'-dissubstituídas, estericamente impedidas.

\section{Reações de ciclo-funcionalização}

A utilização de reações de ciclo-funcionalização, apesar dos altos rendimentos, torna-se restrita pela complexidade dos precursores da ciclização. Mas, para a formação de derivados 2,2'bipiridínicos não-simétricos, particularmente substituídos, esta rota sintética pode ser a única viável. 
Reações de funcionalização de 2,2'-bipiridina e derivados

A funcionalização da 2,2'-bipiridina ou da N,N'-dióxido2,2'-bipiridina correspondente torna-se via preferencial para síntese de 2,2'-bipiridinas 5,5' - e 4,4'-dissubstituídas respectivamente, por reações de substituição eletrofílica aromática usuais. Além do mais a 2,2'-bipiridina é reagente disponível e de baixo custo e as reações nas quais está envolvida também são geralmente comuns, de fácil execução e de bons rendimentos. O derivado N,N'-dióxido permite a execução de transformações de grupo funcional específicas, principalmente para a formação de derivados 6,6'- e 4,4'-dissubstituídos que aumentam o escopo e a aplicabilidade deste método. Cabe ressaltar também que a preparação do derivado mono-N-óxido-2,2'-bipiridina, por oxida- ção seletiva, e a execução de reações subseqüentes, podem levar à formação de derivados bipiridínicos não-simétricos.

Em geral, a formação de derivados 2,2'-bipiridínicos halogenados de diferentes padrões de substituição é importante pois, pode ser seguida por reações de substituições nucleofílicas aromáticas, ou por reações de substituição eletrofílicas aromáticas efetuadas por litiação (com reagente organo-lítio) seguida de adição de agentes eletrofílicos diversos podendo-se sintetizar muitos outros derivados 2,2'-bipiridínicos substituídos diferentes.

Finalmente, a Tabela 1 mostra de forma resumida, os métodos utilizados para a obtenção das 2,2'-bipiridinas substituídas já sintetizadas de grande importância sintética, com as respectivas referências que citam a sua preparação. Estes dados são fornecidos em or-

Tabela 1. Métodos de preparação de 2,2'-bipiridinas substituídas

\begin{tabular}{|c|c|c|c|c|c|}
\hline No. & Substituintes & Ref. & No. & Substituintes & Ref. \\
\hline 1 & $\mathrm{X}=\mathrm{Y}=\mathrm{H}^{\mathrm{a}}$ & $5-13$ & $27^{\mathrm{a}}$ & $\mathrm{X}=\mathrm{H} ; \mathrm{Y}=5-\mathrm{NH}_{2}$ & 30 \\
\hline $3 e$ & $\mathrm{X}=\mathrm{Y}=4-\mathrm{Me}^{\mathrm{a}}$ & $10,19,27$ & $27 b$ & $\mathrm{X}=\mathrm{Y}=5-\mathrm{NH}_{2}$ & 30 \\
\hline $\mathbf{3 d}$ & $\mathrm{X}=\mathrm{Y}=3-\mathrm{Me}$ & $10,24,27$ & 28 & $\mathrm{X}=\mathrm{Y}=5-\mathrm{CN}^{2}$ & 30 \\
\hline $\mathbf{3 f}$ & $\mathrm{X}=\mathrm{Y}=5-\mathrm{Me}$ & 10,27 & 32 & $\mathrm{X}=\mathrm{Y}=6-\mathrm{CN}$ & 36,37 \\
\hline $3 g$ & $\mathrm{X}=\mathrm{Y}=6-\mathrm{Me}$ & $11,19,26$ & $30 \mathbf{a}$ & $\mathrm{X}=\mathrm{Y}=4-\mathrm{COCl}$ & 33 \\
\hline $3 \mathbf{h}$ & $X=Y=4-E t$ & 12 & 30b & $\mathrm{X}=\mathrm{Y}=4-\mathrm{CO}_{2} \mathrm{R}$ & 33 \\
\hline $14 \mathbf{a}$ & $\mathrm{X}=\mathrm{H} ; \mathrm{Y}=4-\mathrm{Me}$ & 25 & $38 \mathrm{~h}$ & $\mathrm{X}=\mathrm{H}, \mathrm{Y}=4-\mathrm{CONH}_{2}^{2}$ & 44 \\
\hline $14 b$ & $\mathrm{X}=\mathrm{H} ; \mathrm{Y}=5-\mathrm{Me}$ & 25 & 44 & $\mathrm{X}=\mathrm{Y}=6-\mathrm{CSNH}_{2}{ }^{\mathrm{b}^{2}}$ & 47,32 \\
\hline $14 c$ & $\mathrm{X}=\mathrm{H} ; \mathrm{Y}=6-\mathrm{Me}$ & 25 & 30c & $\mathrm{X}=\mathrm{Y}=4-\mathrm{CONHOH}$ & 32 \\
\hline $3 \mathbf{i}$ & $\mathrm{X}=\mathrm{Y}=4-\mathrm{C}_{6} \mathrm{H}_{5}$ & 12 & 30d & $\mathrm{X}=\mathrm{Y}=4-\mathrm{COSR}$ & 34 \\
\hline 8 & $\mathrm{X}=\mathrm{Y}=6-\mathrm{C}_{6} \mathrm{H}_{5}$ & 23 & $41 \mathrm{a}$ & $\mathrm{X}=\mathrm{H}, \mathrm{Y}=6-\mathrm{CSNH}_{2}$ & 46 \\
\hline $38 f$ & $\mathrm{X}=\mathrm{H} ; \mathrm{Y}=4-\mathrm{Cl}$ & 44 & $41 b$ & $\mathrm{Y}=4-\mathrm{Cl}, 6-\mathrm{CSNH}_{2}$ & 45 \\
\hline $38 \mathrm{~g}$ & $\mathrm{X}=\mathrm{H} ; \mathrm{Y}=4-\mathrm{Br}$ & 44 & $41 \mathrm{c}$ & $\mathrm{X}=\mathrm{NO}_{2}, \mathrm{Y}=6-\mathrm{CSNH}_{2}$ & 45 \\
\hline $3 \mathbf{a}^{\circ}$ & $\mathrm{X}=\mathrm{Y}=5-\mathrm{Br}$ & $10,30,31$ & 41d & $\mathrm{Y}=4-\mathrm{OMe}, 6-\mathrm{CSNH}_{2}$ & 45 \\
\hline $\mathbf{3 b}$ & $\mathrm{X}=\mathrm{Y}=5-\mathrm{Cl}$ & 10 & $41 e$ & $\mathrm{X}=\mathrm{Y}=4-\mathrm{Me}, \mathrm{Y}=6-\mathrm{CSNH}_{2}$ & 45 \\
\hline $6 \mathbf{a}$ & $\mathrm{X}=\mathrm{H} ; \mathrm{Y}=6-\mathrm{Br}$ & 21,22 & $41 f$ & $\mathrm{X}=\mathrm{Y}=4-\mathrm{OMe}, \mathrm{Y}=6-\mathrm{CSNH}_{2}^{2}$ & 45 \\
\hline $\mathbf{6 b}$ & $\mathrm{X}=\mathrm{Y}=6-\mathrm{Br}$ & 21,22 & $41 \mathrm{~g}$ & $\mathrm{X}=\mathrm{Y}=4-\mathrm{Cl}, \mathrm{Y}=6-\mathrm{CSNH}_{2}{ }^{2}$ & 45 \\
\hline 34 & $\mathrm{X}=\mathrm{Y}=4-\mathrm{Cl}$ & 39,40 & $36 \mathbf{a}$ & $\mathrm{X}=\mathrm{Y}=\mathrm{SR}(\mathrm{Et}, \mathrm{Me})^{2}$ & 39 \\
\hline $3 c$ & $\mathrm{X}=\mathrm{Y}=5-\mathrm{Cl}$ & 10 & $38 b$ & $\mathrm{X}=\mathrm{H}, \mathrm{Y}=\mathrm{SBz}$ & 44 \\
\hline $\mathbf{4 a}$ & $\mathrm{X}=\mathrm{Y}=6-\mathrm{Cl}$ & 18 & 36b & $\mathrm{X}=\mathrm{Y}=\mathrm{SO}_{3} \mathrm{H}$ & 39 \\
\hline $11 a$ & $\mathrm{X}=\mathrm{H} ; \mathrm{Y}=3-\mathrm{CO}_{2} \mathrm{Me}$ & 22 & $37 \mathbf{a}$ & $\mathrm{X}=\mathrm{Y}=\mathrm{OR}$ & 38,41 \\
\hline $11 b$ & $\mathrm{X}=\mathrm{H} ; \mathrm{Y}=4-\mathrm{CO}_{2}^{2} \mathrm{Me}$ & 22 & $38 \mathrm{e}$ & $\mathrm{X}=\mathrm{H}, \mathrm{Y}=\mathrm{OBz}$ & 44 \\
\hline $11 \mathrm{c}$ & $\mathrm{X}=\mathrm{H} ; \mathrm{Y}=5-\mathrm{CO}_{2} \mathrm{Me}$ & 22 & 38c,d & $\mathrm{X}=\mathrm{H}, \mathrm{Y}=\mathrm{OR}(\mathrm{Et}, \mathrm{Me})$ & 44 \\
\hline 11d & $\mathrm{X}=\mathrm{H} ; \mathrm{Y}=6-\mathrm{CO}_{2}^{2} \mathrm{Me}$ & 22 & $37 \mathbf{b}$ & $\mathrm{X}=\mathrm{Y}=\mathrm{NR}$ & 38,41 \\
\hline $11 e$ & $\mathrm{X}=\mathrm{H} ; \mathrm{Y}=3-\mathrm{CO}_{2}^{2} \mathrm{H}$ & 22 & $38 b$ & $\mathrm{X}=\mathrm{H}, \mathrm{Y}=4-\mathrm{NH}_{2}$ & 44 \\
\hline $11 f$ & $\mathrm{X}=\mathrm{H} ; \mathrm{Y}=4-\mathrm{CO}_{2} \mathrm{H}$ & 22,32 & 33 & $\mathrm{X}=\mathrm{Y}=4-\mathrm{NO}_{2}$ & 32,38 \\
\hline $11 \mathrm{~g}$ & $\mathrm{X}=\mathrm{H} ; \mathrm{Y}=5-\mathrm{CO}_{2}^{2} \mathrm{H}$ & $14,22,2132$ & $38 \mathrm{i}$ & $\mathrm{X}=\mathrm{H}, \mathrm{Y}=4-\mathrm{NO}^{2}$ & 30 \\
\hline $11 \mathrm{~h}$ & $\mathrm{X}=\mathrm{H} ; \mathrm{Y}=6-\mathrm{CO}_{2}^{2} \mathrm{H}$ & 22,21 & $39 \mathbf{a}$ & $\mathrm{X}=4-\mathrm{Cl}, \mathrm{Y}=4-\mathrm{NO}_{2}^{2}$ & 42 \\
\hline 29 & $\mathrm{X}=\mathrm{Y}=3-\mathrm{CO}_{2} \mathrm{H}$ & 30 & $39 \mathrm{~b}$ & $\mathrm{X}=4-\mathrm{OMe}, \mathrm{Y}=4-\mathrm{NO}_{2}^{2}$ & 42 \\
\hline $35 \mathbf{a}$ & $\mathrm{X}=\mathrm{Y}=3-\mathrm{CHO}$ & 21 & 42 & $\mathrm{X}=\mathrm{Y}=4-\mathrm{Me}-$ tiazol-2il & 47 \\
\hline 52 & $\mathrm{X}=\mathrm{H}, \mathrm{Y}=4-\mathrm{CHO}$ & 54 & 43 & $\mathrm{X}=\mathrm{Y}=4$-py-tiazol-2il & 47 \\
\hline 53 & $\mathrm{X}=\mathrm{Y}=4-\mathrm{CHO}$ & 55 & 45 & $\mathrm{X}=\mathrm{Y}=4-\mathrm{CH}_{2} \mathrm{Cl}$ & 48,52 \\
\hline $35 b$ & $\mathrm{X}=\mathrm{Y}=4-\mathrm{COMe}$ & 21 & 46 & $\mathrm{X}=\mathrm{Y}=4-\mathrm{CH}_{2}^{2} \mathrm{Br}$ & $50,52,53$ \\
\hline $11 \mathrm{i}$ & $\mathrm{X}=\mathrm{H} ; \mathrm{Y}=3-\mathrm{CH}_{2} \mathrm{OH}$ & 22 & 47 & $\mathrm{X}=\mathrm{Y}=4-\mathrm{CH}_{2} \mathrm{SiMe}_{3}$ & 52 \\
\hline $11 \mathbf{j}$ & $\mathrm{X}=\mathrm{H} ; \mathrm{Y}=4-\mathrm{CH}_{2}^{2} \mathrm{OH}$ & 22 & 48 & $\mathrm{X}=\mathrm{Y}=4-\mathrm{CH}_{2} \mathrm{CHOHBn}$ & 52 \\
\hline 111 & $\mathrm{X}=\mathrm{H} ; \mathrm{Y}=5-\mathrm{CH}_{2} \mathrm{OH}$ & 22 & 49 & $\mathrm{X}=\mathrm{Y}=4-\mathrm{CH}_{2} \mathrm{OAc}$ & 53 \\
\hline $11 \mathrm{~m}$ & $\mathrm{X}=\mathrm{H} ; \mathrm{Y}=6-\mathrm{CH}_{2} \mathrm{OH}$ & 22 & 50 & $\mathrm{X}=\mathrm{Y}=4-\mathrm{CH}_{2} \mathrm{OH}$ & 53 \\
\hline $11 n$ & $\mathrm{X}=\mathrm{H} ; \mathrm{Y}=3-\mathrm{CH}_{2}^{2} \mathrm{NH}_{2}$ & 22 & $51 a$ & $\mathrm{X}=\mathrm{Y}=4-\mathrm{CH}_{2} \mathrm{Bz}$ & 52 \\
\hline 110 & $\mathrm{X}=\mathrm{H} ; \mathrm{Y}=4-\mathrm{CH}_{2} \mathrm{NH}_{2}$ & 22 & $51 b$ & $\mathrm{X}=\mathrm{Y}=4-\mathrm{CH}_{2} \mathrm{C}_{12} \mathrm{H}_{25}$ & 52 \\
\hline $11 p$ & $\mathrm{X}=\mathrm{H} ; \mathrm{Y}=5-\mathrm{CH}_{2} \mathrm{NH}_{2}^{2}$ & 22 & $51 \mathrm{c}$ & $\mathrm{X}=\mathrm{Y}=4-\mathrm{CH}_{2} \mathrm{CH}_{2} \mathrm{CN}$ & 52 \\
\hline $11 q$ & $\mathrm{X}=\mathrm{H} ; \mathrm{Y}=6-\mathrm{CH}_{2} \mathrm{NH}_{2}$ & 22 & 54 & $\mathrm{X}=\mathrm{Y}=\mathrm{CH}=\mathrm{CH}_{2}$ & 55 \\
\hline $38 b$ & $\mathrm{X}=\mathrm{H} ; \mathrm{Y}=4-\mathrm{NH}_{2}$ & 44 & 55 & $\mathrm{X}=\mathrm{Me}, \mathrm{Y}=\mathrm{CH}=\mathrm{CH}_{2}$ & 56 \\
\hline
\end{tabular}

${ }^{\mathrm{a}}$ Disponível comercialmente; ${ }^{\mathrm{b}} \mathrm{o}$ derivado $\mathrm{CONH}_{2}$ também foi preparado 
dem de complexidade e similaridade da substituição a fim de proporcionar um guia fundamental, prático e objetivo, para pesquisa sobre os métodos de síntese descritos para a preparação destes compostos de tão grande importância e aplicabilidade.

\section{AGRADECIMENTOS}

Os autores agradecem ao CNPq, à FAPEMIG, à FUNDEPUFMG, à FINEP, ao PADCT e à Pró- Reitoria de Graduação-UFMG pelo apoio financeiro e pelas bolsas concedidas. Agradecemos também à Profa. Dra. Maria Helena de Araújo pelas valiosas sugestões.

\section{REFERÊNCIAS}

1. Tenenbaum, L. E., Em Heterocyclic Compounds, Klingsberg, E., ed., Interscience Publishers. Inc.: New York, 1961, vol. 2, p. 224-228 Frankenburg W. G., Gottscho A. M., Mayaud E. W.; J. Am. Chem. Soc. 1952, 74, 4309 .

2. Wilkinson, G. Em Comprehensive Coordination Chemistry, Gillard, R.; Mcleverty, J. A., eds.; Pergamon Press: Oxford, 1985, vol. 2, p. 73.

3. http://www.webofscience.com, acessada em Outubro de 2001.

4. Kaes, C.; Katz, A.; Hosseini, M. W.; Chem. Rev. 2000, 100, 3553.

5. Blau, F.; Monatsh 1898, 19, 647 .

6. Leffler, M. T.; Organic Reactions 1942, $1,19$.

7. Tokyana; J. Pharm. Soc. Japan 1954, 74,1404.

8. Fanta, P. E.; Chem. Ber. 1946, $38,139$.

9. Case, F. H.; J. Chem. Soc. 1961, 2574.

10. Wibaut, J. P.; Overhof, R.; Recl. Trav. Chim. 1928, 47, 761.

11. Willink, T. H. D.; Wibaut, J. P.; Recl. Trav. Chim. 1935, 54, 275.

12. Case, F. H.; Kasper, T. J.; J. Am. Chem. Soc. 1956, 78, 5842.

13. Hein, F.; Retter, R. H.; Ber. 1928, 61, 1790.

14. Sasse, W. H. F.; Whittle, C. P.; J. Chem. Soc. 1961, 1347; Badger, G. M.; Sasse, W. H. F.; J. Am. Chem. Soc. 1956, 616; Wibaut, J. P.; Overhof, R.; Recl. Trav. Chim. $1928,50,287$.

15. Wibaut, J. P.; Willink; T. H. D.; Recl. Trav. Chim. 1931, 50 , 287; 1935 , 54,$804 ; \mathbf{1 9 4 3}, 62,466$

16. Burstall, F. H.; J. Chem. Soc. 1938, 1662.

17. Rosevear, P. E.; Sasse, W. H. F.; J. Heterocycl. Chem. 1971, 8, 483.

18. Newkome, G. R.; Hager, C. D.; J. Am. Chem. Soc. 1978, 100, 5567.

19. Uchida, Y.; Kozawa, H.; Tetrahedron Lett. 1989, 30, 6365.

20. Oae, S.; Inubushi, Y.; Yioshihara, M.; Phosphorus Sulfur ; 1995, 103, 101

21. Parks, J. E.; Wagner, B. E.; Holm, R. H.; J. Organomet. Chem. 1973, 56, 53.

22. Garber, T.; Rillema, D. P.; Synth. Commun. 1990, 20, 1233.

23. Leadbeater, N. E.; Resoule, S. M.; Tetrahedron Lett. 1999, 40, 4243.

24. Schubert, U. S. , Eschbaumer, C.; Hochwimmer, G.; Synthesis 1999, 779; Panetta, C. A.; Kumpaty, H. J.; Heimer, N. E.; Leavy, M. C. ; Hussey, C. L.; J. Org. Chem. 1999, 64, 1015.

25. Savage, S. A.; Smith, A. P.; Fraser, C. L.; J. Org. Chem. 1998, 63, 10048.

26. Navarro, M.; Cassol, T. M.; Demintz, F. W. J.; Neves, E. A. D.; Tetrahedron Lett. 2000, 41,8203 .
27. Navarro, M. ; França, K. W. R.; Demintz, F. W. J.; Leonel, E.; Nédeléc, J. Y.; XII Simpósio Brasileiro de Eletroquímica e Eletroanalítica, Gramado, Brasil, 2001

28. Joule, J. A.; Mills, K.; Smith, G. F.; Heterocyclic Chemistry, 3ㄹ ed., Londres: Reino Unido, 1995.

29. Potts, K. T.; Winslow, R.; J. Org. Chem. 1985, 50, 5405; Potts, K. T.; Rayford, G. K.; Keshavarz, M.; J. Org. Chem 1993, 115, 2795.

30. Morgan, R.; Burstall, F. H.; J. Chem. Soc. 1934, 1498; 1937, 1649.

31. Romero, F. M.; Ziessel, R.; Tetrahedron Lett. 1995, 36, 6471.

32. Donnici, C. L.; Máximo, D. H.;Moreira, L. L. C.; Reis, G. T.; Cordeiro, E. S.; Oliveira, I. M. F.; Carvalho, S.; Paniago, E.; J. Braz. Chem. Soc. 1998, 9, 455

33. Sprintschnik, G.; Sprintschnik, H. W.; Kirsch, P. P.; Whitten, D. G.; J. Am. Chem. Soc. 1977, 99, 4947.

34. Donnici, C.L.; Oliveira, I. M. F.; trabalho não publicado.

35. Haginiwa, J.; J. Pharm. Soc. Japan 1955, 75, 731.

36. Baxter, P. N. W.; Connor, J.A.; Schweizer, W. B.; Wallis, J. D.; J. Chem. Soc. Dalton Trans. 1992, 3015.

37. Antonini, I.; Cristalli, G.; Franchetti, P.; Grifantini, M.; Martelli, S. Il Farmaco 1986, 41, 345.

38. Maerker, G.; Case F. H.; J. Am. Chem. Soc. 1958 , 80, 2745; Case, F.H.; J. Am. Chem. Soc., $1946,68,2574$.

39. Wenkert, D.; Woodward, R. B.; J .Org. Chem. 1983, 48, 283.

40. Anderson, S.; Constable, E. C.; Seddon, K. R.; Turp, J. E.; Baggott, J. E.; Pilling, M. J.; J. Chem. Soc. Dalton Trans. 1985, 2247.

41. Connor, J. A.; Overton, C.; J. Organomet. Chem. 1983, 249, 165.

42. Cook, M. J.; Lewis, A. P.; McAuliffe, G. S. G.; Skarda, V.; Thomson, A. J.; Glasper, J. L. Robbins, D. J.; J. Chem. Soc. Perkin Trans. 1984, 1293.

43. Demnitz, F. W. J.; Hemi, M. B.; Org. Proced. Int. 1998, 30, 467.

44. Jones, R. A.; Roney, B. D.; Sasse, W. F. H.; Wade, K. O.; J. Chem. Soc. B 1967, 106.

45. Antonini, I.; Cristalli, G.; Franchetti, P.; Grifantini, M.; Martelli, S.; Il Farmaco 1986, 41, 345.

46. Antonini, I.; Claudi, F.; Cristalli, G.; Franchetti, P.; Grifantini, M.; Martelli, S.; J. Med. Chem. 1981 , 24, 1181.

47. Connor, P. N. W.; B axter, J. A.; Schweizwer, W. B.; Wallis, J. D.; J. Chem. Soc. Dalton Trans 1992, 3015.

48. Newkome, G. R.; Kieffer, G. E.; Xia, Y. J.; Gupta, V.K.; Synthesis 1984, 677

49. Newkome, G. R.; Puckett, W. E.; Kieffer, G. E.; Xia, Y. J.; Gupta, V.K.; Coreil, M.; Hackney, M. A.; J. Org. Chem. 1982, 47, 4116.

50. Gould, S.; Strouse, G. F.; Meyer, T. J.; Sullivan, B. P.; Inorg. Chem. 1991, 30, 2942.

51. Uenishi, J.; Tanaka, T.; Nishiwaki, K.; Wabayashi, S.; Oae, S.; Tsukube, H.; J. Org. Chem. 1993, 58, 4382.

52. Fraser, C. L.; Anastasi, N. R.; Lamba, J. S.; J. Org. Chem. 1997, $62,9314$.

53. Fraser, C. L.; Corbin, P. S.; Smith, A. P.; Tetrahedron Lett. 2000, 41, 2787.

54. Oivannen, M.; Mikhailov, S. N.; Padyukova, N. S.; Lönnberg, H.; J. Org. Chem. 1993, 58, 1617.

55. Della Ciana, L.; Dressick, W. J.; Zelewsky, A.; J. Heterocycl. Chem. 1990, 27, 163.

56. Ghosh, P. K.; Spiro, T. G.; J .Am. Chem. Soc. 1980, 102, 5543. 